American Journal of Applied Sciences 5 (12): 1804-1807, 2008

ISSN 1546-9239

(C) 2008 Science Publications

\title{
Small Size and Dual Band of a Quadratic Koch Dipole Fractal Antenna Design
}

\author{
Fawwaz J. Jibrael, Faez F. Shareef and Wafaa S. Mummo \\ Department of Electrical and Electronic Engineering, University of Technology, \\ P.O. Box 35010, Baghdad, Iraq
}

\begin{abstract}
The performance and analysis of a small size, low profile and dual band quadratic Koch curve dipole antenna was introduced. The proposed antenna design, analysis and characterization had been performed using the method of moments $(\mathrm{MoM})$ technique. The radiation characteristics, VSWR, reflection coefficient and input impedance of the proposed antenna were described and simulated using 4NEC2 software package, MATLAB v7.2 and NEC viewer. From the simulated results, the proposed antenna has an operating frequency of $459 \mathrm{MHz}$ and $1261 \mathrm{MHz}$ and at these frequencies the voltage stand wave ratio (VSWR) less than from 2.
\end{abstract}

Key words: Koch curve, fractal antenna, dual band antenna, multiband antenna

\section{INTRODUCTION}

As part of an effort to further improve modern communication system technology, researchers are now studying many different approaches for creating new and innovative antennas. One technique that has received much recent attention involves combining aspects of the modern theory of fractal geometry with antenna design ${ }^{[1]}$. Fractals were first defined by Mandelbrot $^{[2]}$ as a way of classifying complex geometric structures that have non-integer dimensionality and which posses' inherent selfsimilarity or self-affinity with-in their geometrical structure. While Euclidean geometries are limited to points, lines, sheets and volumes of integer dimensionality, fractal structures fall between these Euclidean classifications having non-integer dimensionality. Fractal geometries accurately characterize many non-Euclidean features of the natural including the length of coastline, density of clouds and the branching of trees ${ }^{[3]}$ and find application in many areas of science and engineering including antenna design. Nowadays, the design of multiband and small size antennas is still of major importance as an engineer topic. The growths of the telecommunication systems are driving the engineering efforts to develop multiservice (multiband) and compact (portable) systems which require such kind of antennas. In case of cellular systems, not only the handset antenna is important, but also those on base stations. It is in this framework where fractal technology appears potentially as powerful tool to meet the telecommunication operator requirements ${ }^{[4]}$. Fractal shaped antennas are becoming a useful way to design advanced antennas such as multiband antennas with approximately the same input or radiation characteristics for different frequency bands. This comes out from the fact that most of the fractals are selfsimilar objects. That is roughly speaking fractals composed many copies of themselves at different scales. The global fractal form is repeated at different sizes as many times as desired within the object structure such that the global object and its parts become identical ${ }^{[4]}$. In addition to the simplicity and self-similarity, fractal curves have the additional property of approximately filling a plane which makes them the attractive candidates for use in the design of antennas ${ }^{[5]}$. An example of plane filling Hilbert curve had been demonstrated in ${ }^{[6]}$, also the Koch monopole and dipole had been demonstrated in ${ }^{[7]}$. This paper presents the design and simulation a wire dipole antenna based on the first iteration quadratic Koch curve geometry.

\section{PROPOSED ANTENNA GEOMETRY}

Figure 1 contains the first three iterations in the construction of the quadratic Koch curve, also known as the Minkowski sausage. This curve is generated by repeatedly replacing each line segment, composed of four quarters, with the generator consisting of eight pieces, each one quarter long (Fig. 1) ${ }^{[8]}$. Each smaller segment of the curve is an exact replica of the whole curve.There are eight such segments making up the curve, each one a one-quarter reduction of the original

Corresponding Author: Fawwaz J. Jibrael, Department of Electrical and Electronic Engineering University of Technology, P.O. Box 35010, Baghdad, Iraq 


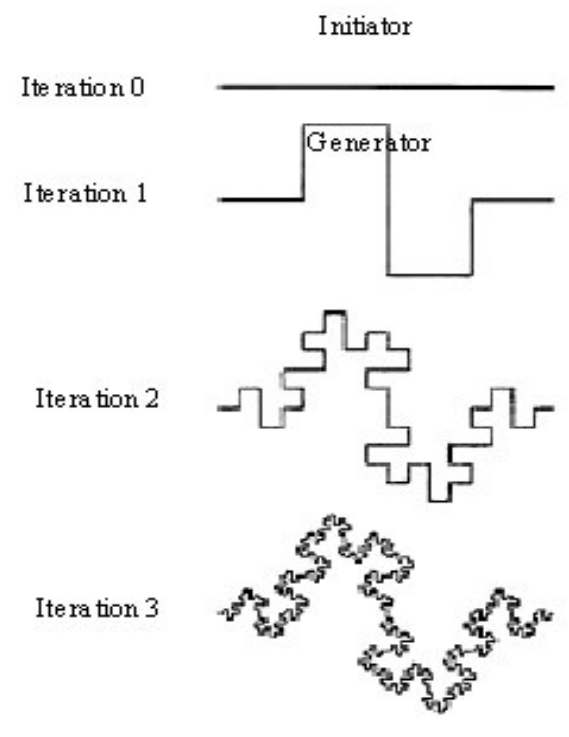

Fig.1: First three iterations of the construction of the quadratic Koch curve.

curve. Thus, $\mathrm{N}=8, \varepsilon=1 / 4$ and the fractal similarity dimension (FD) is:

$$
\mathrm{FD}=\frac{\log (\mathrm{N})}{\log (1 / \varepsilon)}=\frac{\log (8)}{\log (4)}=1.5
$$

Where $\mathrm{N}$ is the total number of distinct copies and $(1 / \varepsilon)$ is the reduction factor value which means how will be the length of the new side with respect to the original side length.

Figure 2 shows the first iteration of quadratic Koch curve dipole antenna. The antenna design and simulation have been performed using the 4NEC2 package.

\section{MATHEMATICAL MODELING}

The numerical simulations of the antenna system are carried out via the method of moments. Numerical modeling commercial software 4NEC2 is used in all simulations. The NEC is a computer code based on the method of moment for analyzing the electromagnetic response of an arbitrary structures consisting of wires or surfaces, such as Hilbert and Koch curves. The modeling process is simply done by dividing all straight wires into short segments where the current in one segment is considered constant along the length of the short segment. It is important to make each wire segment as short as possible without violation of maximum segment length to radius ratio computational restrictions. In NEC, to modeling a wire structures, the

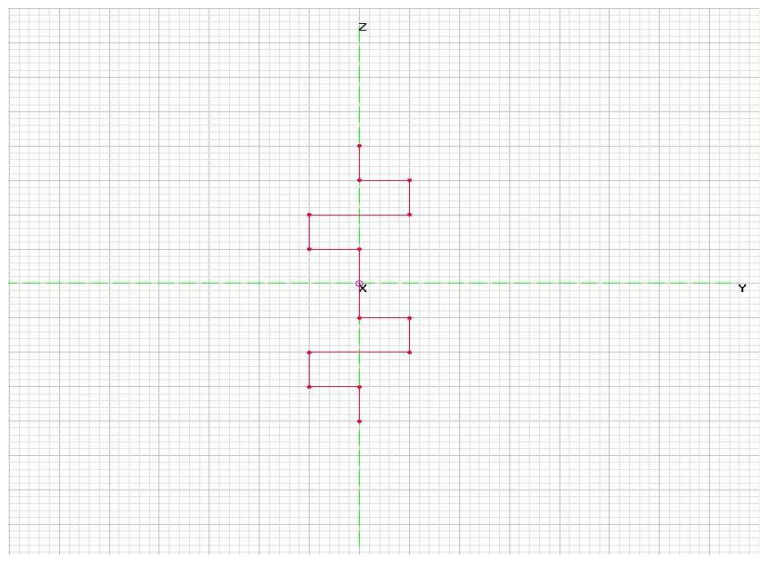

Fig. 2: Quadratic Koch curve dipole antenna

segments should follows the paths of conductor as closely as possible ${ }^{[9]}$.

\section{ANTENNA DESIGN}

The quadratic Koch antenna shown in Fig. 2 which positioned in the YZ-plane has been simulated using numerical modeling commercial software 4NEC2, which is method of moment based software. The method of moment (MoM) is used to calculate the current distribution along the quadratic Koch curve and hence the radiation characteristics of the antenna ${ }^{[10]}$. The 4NEC2 program is used in all simulations. This is very effective in analyzing antennas that can be modeled with wire segments, such as the one under consideration here. To suit the requirements, the antenna is modeled without any dielectric present, although some of the practical implementations do require dielectric support ${ }^{[6]}$.

The feed source point of this antenna is placed at origin $(0,0,0)$ and this source set at 1 volt. The design frequency has been chosen to be $750 \mathrm{MHz}$ which give design wavelength $\lambda$ is $0.4 \mathrm{~m}(40 \mathrm{~cm})$ then the length of the corresponding $\lambda / 2$ dipole antenna length will be of $20 \mathrm{~cm}$ and the conductor diameter has been chosen to be $1 \mathrm{~mm}$, as shown in Fig. 2 .

Figure 3 shows the visualization of this dipole antenna geometry by using NEC-viewer software.

\section{RESULTS AND DISCUSSION}

The real and imaginary parts of the input impedance of this proposed antenna are shown in Fig. 4 over a frequency range from $0 \mathrm{GHz}-3 \mathrm{GHz}$. The input impedances characteristics of this proposed antenna show that this antenna has dual band frequencies. 


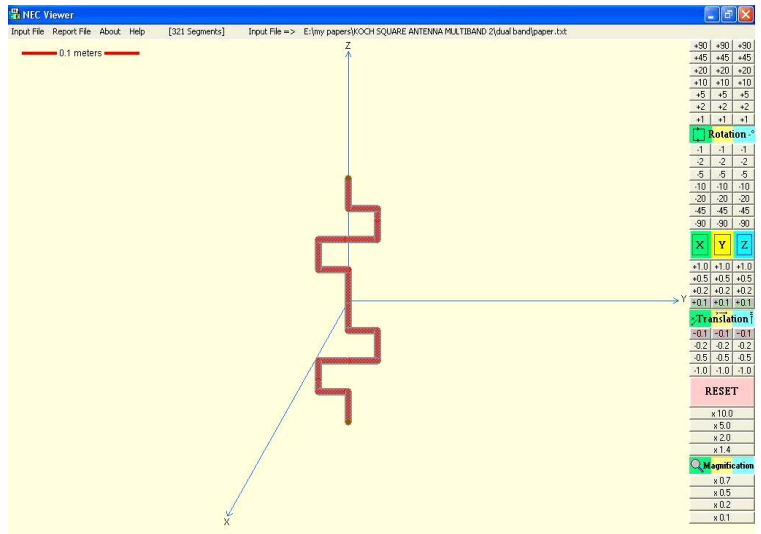

Fig. 3: Visualization of the modeled dipole antenna geometry

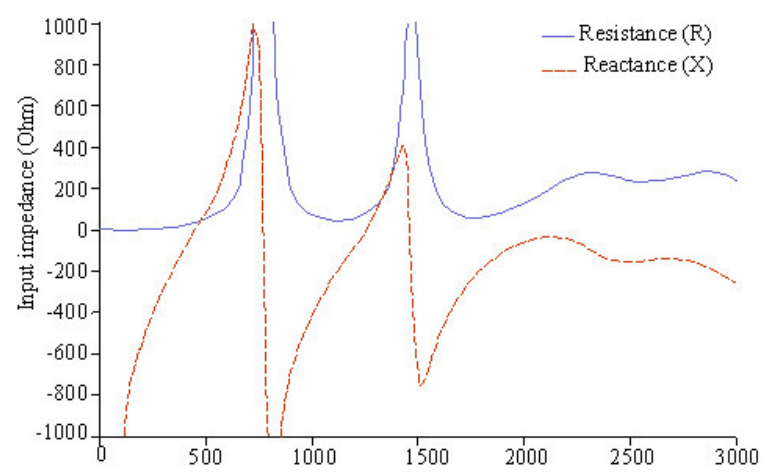

Fig. 4: Input impedance characteristics of the quadratic Koch curve antenna

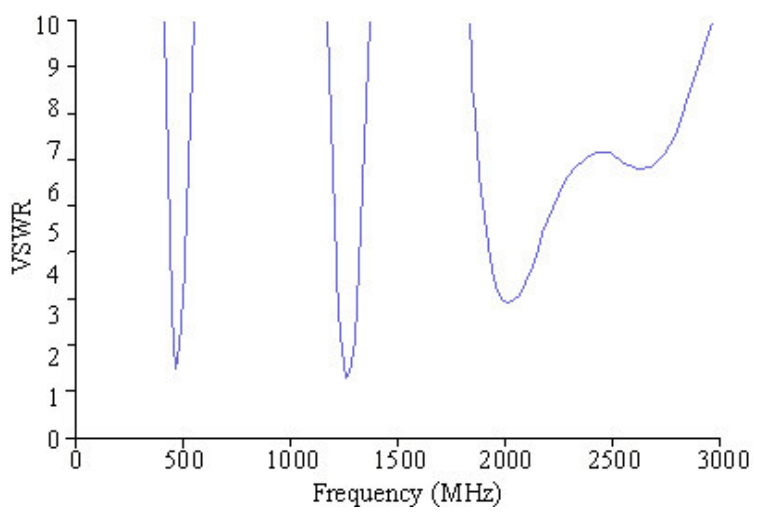

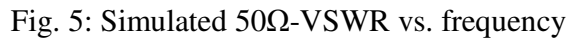

VSWR of the antenna is shown in Fig. 5. It is found that the antenna has dual band behavior at the resonance frequencies $459 \mathrm{MHz}$ and $1261 \mathrm{MHz}$ and at these frequencies VSWR $<2$.

Table 1: Resonant frequencies and input impedance for proposed antenna

\begin{tabular}{lllll}
\hline $\begin{array}{l}\text { Frequency } \\
(\mathrm{MHz})\end{array}$ & $\mathrm{Input}$ impedance $(\Omega)$ & & $\begin{array}{l}\text { Reflection } \\
\text { coefficient }(\mathrm{dB})\end{array}$ \\
\hline 459 & $\mathrm{R}$ & $\mathrm{X}$ & $\mathrm{VSWR}$ & -11.7 \\
1261 & 29.38 & $\mathrm{j} 0.026$ & 1.702 & -14.9 \\
\hline
\end{tabular}

Table 1 shows these resonant frequencies, VSWR, reflection coefficient and the corresponding input impedances of each one.

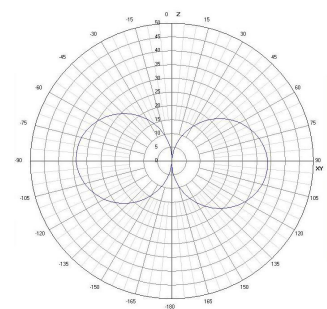

YZ-plane

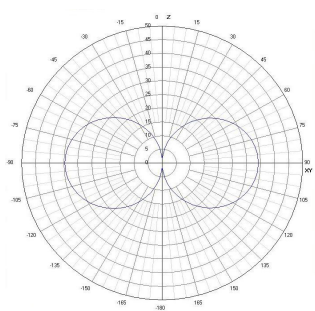

XZ-plane

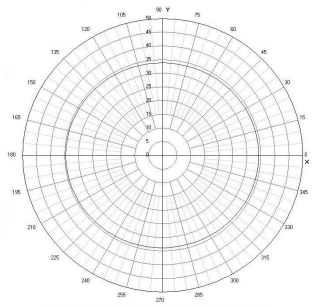

XY-plane

(a) $\mathrm{f}=459 \mathrm{MHz}$

Fig.6: Radiation patterns of the proposed antenna at resonant frequencies of 459 , and $1261 \mathrm{MHz}$.

The radiation patterns at these resonant frequencies in the planes YZ-plane, XZ-plane and XY-plane have been demonstrated as in Fig.6, where the antenna is placed in the YZ-plane. 


\section{CONCLUSIONS}

In this research, the quadratic Koch curve dipole antenna based on the first iteration has been investigated and its performance has been evaluated. The Simulation results show that this antenna can be efficiently operates as a dual band antenna and has a compact size property. The proposed antenna has two resonating bands at frequencies of $459 \mathrm{MHz}$ and $1261 \mathrm{MHz}$. at these frequencies this antenna have VSWR $<2$.

According to these frequencies, this antenna can operate as a multiband antenna in the UHF applications. Once optimized for radiation characteristics, this antenna can find many applications in UHF communication systems.

\section{REFFERENCES}

1. Petko, S.J. and D. Werner, 2004. Miniature reconfigurable three-dimensional fractal tree antennas. IEEE Trans. Antennas Propagat., 52:1945-1956.

http://ieeexplore.ieee.org/xpl/freeabs_all.jsp?arnum ber $=1321324$

2. Falconer, K., 1990. Fractal Geometry: Mathematical Foundation and Applications. $2^{\text {nd }}$ Edn., John Wiley, England.

3. Werner, D.H. and S. Ganguly, 2003. An overview of fractal antennas engineering research. IEEE Antennas Propagat. Mag., 45: 38-56. http://ieeexplore.ieee.org/Xplore/login.jsp?url=/iel5 /74/26673/01189650.pdf?temp $=\mathrm{x}$

4. Wemer, D. and Raj Mitra, 2000. Frontiers in Electromagnetics. IEEE Press Series on Microwave Technology and RF, $2^{\text {nd }}$ Edn. New York.
5. Gianvitorio, J. and Y. Rahmat, 2002. Fractal antennas: A novel antenna miniaturization technique and applications. IEEE Antennas Propagat.Mag.,44:20-36.

http://ieeexplore.ieee.org/Xplore/login.jsp?url=/iel5 174/21527/00997888.pdf?temp $=\mathrm{x}$

6. Vinoy, K.J., K.A. Jose, V.K. Varadan and V.V. Varadan, 2001. Hilbert curve fractal antenna: A small resonant antenna for VHF/UHF applications. Microwave Optical Technol. Lett., 29:215-219.

http://www3.interscience.wiley.com/journal/78505 862/abstract?CRETRY $=1 \&$ SRETRY $=0$

7. Zainud-Deen, S.H., K.H. Awadalla, S.A. Khamis and N.D. El-shalaby, 2004. Radiation and scattering from koch fractal antennas. 21st National Radio Science Conference (NRSC), March 16-18, B8-1-9.

8. Paul, S. Addison, 1997. Fractals and Chaos: An Illustrated Course. Institute of Physics Publishing Bristol and Philadelphia.

9. Burke, G.J. and A.J Poggio, 1981. Numerical Electromagnetic Code (NEC) Program Description. Lawrence Livermore Laboratory.

10. Balanis, C.A., 1997. Antenna Theory: Analysis and Design. 2nd Edn., John Wiley and Sons, New York. 